\title{
La philosophie de la culture de Georg Simmel, un humanisme sans anthropologie?
}

\section{Matthieu Amat}

\section{CpenEdition}

\section{Journals}

Édition électronique

URL : http://journals.openedition.org/alter/367

DOI : $10.4000 /$ alter.367

ISSN : 2558-7927

Éditeur :

Association ALTER, Archives Husserl (CNRS-UMR 8547)

\section{Édition imprimée}

Date de publication : 1 novembre 2015

Pagination : 9-27

ISBN : 978-2-9550449-1-9

ISSN : $1249-8947$

\section{Référence électronique}

Matthieu Amat, "La philosophie de la culture de Georg Simmel, un humanisme sans anthropologie? ", Alter [En ligne], 23 | 2015, mis en ligne le 01 décembre 2017, consulté le 19 avril 2019. URL : http:// journals.openedition.org/alter/367; DOI : 10.4000/alter.367 


\section{LA PHILOSOPHIE DE LA CULTURE DE GEORG SIMMEL, UN HUMANISME SANS ANTHROPOLOGIE?}

MEthieu AmEt

\section{Simmel précurseur de l'anthropologie philosophique?}

Deux principes fondamentaux peuvent caractériser le courant de pensée qui, à partir des travaux fondateurs de Max Scheler et de Helmuth Plessner, s'est nommé « anthropologie philosophique » et a voulu proposer, face à l'éclatement des sciences et de la culture, une nouvelle "image de $1^{\prime}$ homme $»^{1}$. Le premier principe est celui de la "réduction anthropologique ": l'ensemble des produits et domaines culturels doivent être considérés à l'aune de leur valeur et de leur fonction pour la vie humaine, dans la mesure où ils sont le résultat de son activité. Réciproquement, la connaissance de l'homme implique la connaissance de ces produits : l'homme ne peut être connu qu'à partir de ses objectivations ${ }^{2}$. L'ensemble des sciences de la culture participe donc du projet de l'anthropologie. Le second est le «principe de l'interprétation anthropologique des phénomènes particuliers de la vie humaine »: les phénomènes spécifiquement humains (position droite, main préhensible, rire, etc.) mais non strictement culturels doivent trouver place dans cette image de l'homme ${ }^{3}$. Les sciences de la nature, telles la biologie ou la paléontologie participent donc également du projet de l'anthropologie, qui manifeste par là son ambition génétique.

\footnotetext{
${ }^{1}$ O. F. Bollnow, « Die philosophische Anthropologie und ihre methodischen Prinzipien », in R. Rocek et O. Schatz, Philosophische Anthropologie heute, Munich, Beck, 1972, p. 33.

${ }^{2}$ Ibid., p. 27.

${ }^{3}$ Ibid., p. 30.
} 
L'histoire des sources de l'anthropologie philosophique devrait vraisemblablement attribuer un rôle significatif à la pensée de Georg Simmel. Ce rôle est parfois suggéré mais jamais précisément déterminé. Plusieurs points de départ s' offrent à qui voudrait préciser cette histoire. On pourrait développer les indications de Bollnow, qui suggère l'importance de Simmel dans une généalogie de l'anthropologie philosophique à partir de la philosophie de la vie, en mettant en avant les thèmes simméliens de la «transcendance immanente » de la vie et surtout de $1^{\prime}$ homme comme être de limite ${ }^{4}$. Une étude précise du rapport de Max Scheler à Simmel serait également utile, qui montrerait tout ce que l'opposition schélerienne de la vie et de l'esprit doit à Simmel. Il serait probablement fécond, par ailleurs, de développer les suggestions de Hans Blumenberg, qui trouve chez le Simmel psychologue et sociologue des motifs anthropologiques plus concrets : l'attention portée aux effets du regard, à l'expérience du visage, à la sociologie des sens, aux phénomènes de proximité et de distance ${ }^{5}$. Plus récemment, Aldo Haesler et Alexis Dirakis ont suggéré de prolonger le programme plessnérien sous la forme d'une anthropologie relationniste et se réfèrent sur ce point explicitement à Simmel ${ }^{6}$.

Ces travaux restent à faire. Toutefois, comme il nous paraît plus fécond de repérer des tensions que des continuités, nous allons plutôt tâcher de souligner ce par quoi la pensée de Simmel paraît la moins compatible avec le projet de l'anthropologie philosophique et même lui résiste, malgré certaines affinités - concernant notamment la mise en avant de l'indétermination de l'être de l'homme. Notre hypothèse est que cette résistance provient d'une orientation humaniste de la pensée de Simmel qui est absente de l'anthropologie philosophique, ou du moins tendra à disparaître après Scheler et Plessner. Ce que nous entendons par humanisme apparaîtra progressivement ; disons pour le moment qu'il s'agit d'appréhender le processus culturel d'objectivation et la relation de l'homme à ses objectivations non

\footnotetext{
${ }^{4}$ O. F. Bollnow, Die Lebensphilosophie, Berlin, Springer, 1958, p. 32-33, 100.

${ }^{5}$ H. Blumenberg, LEdescription de l'homme, Paris, Cerf, 2011, p. 799 et 801-806.

${ }^{6}$ A. Dirakis, A. Haesler, «Topique et métaphysique dans l'acosmie: Max Scheler, Helmuth Plessner et la genèse de l'Anthropologie Philosophique ", in MEx Scheler. Éthique et phénoménologie, G. Maheo et E. Housset (éd.), Rennes, Presses Universitaires de Rennes. On dispose par ailleurs d'un argument fort en faveur de la thèse d'un Simmel précurseur de l'anthropologie en ce fait que Michael Landmann, commentateur historique de Simmel, a été lui-même un acteur important de l'anthropologie philosophique de l'après-guerre et a pu affirmer que «l'anthropologie est le nom même de la philosophie » (cité par Odo Marquard, «Zur Geschichte des philosophischen Begriffs "Anthropologie" seit dem Ende des achtzehnten Jahrhunderts", in Schwierigkeiten mit der Geschichtsphilosophie, Francfort-sur-le-Main, Suhrkamp, 1973, p. 122).
} 
comme les objets d'une connaissance anthropologique possible, mais en tant qu'ils posent des problèmes axiologiques, notamment celui de la culture en tant que formation ${ }^{7}$. Nous montrerons que cet accent mis sur le problème de la valeur conduira Simmel à se désintéresser des questions génétiques et, surtout, à refuser la réduction anthropologique décrite plus haut. Nous verrons ce qui, dans cette résistance à l'anthropologie, éloigne Simmel de Dilthey et le rapproche de Husserl.

\section{La critique relativiste du concept d'homme}

Les termes $\mathrm{d}^{\prime}$ « homme » (Mensch) ou d' « humanité » (Menschheit) ne sont que très rarement l'objet d'une thématisation expresse chez Simmel, à la différence des concepts de vie, d'esprit, de culture, d'individu ou même de personnalité. Aucun article ou essai ne contient le mot «Mensch» dans son titre et l'on trouve dans les œuvres complètes seulement trois développements significatifs consacrés aux concepts d'homme et d'humanité. Le premier est l'occasion d'une critique déconstructrice de ces concepts.

Le jeune Simmel défend, dans les années 1890, une vision du monde "historico-sociologique», mêlant évolutionnisme darwinien et psychologie et sociologie spencériennes (GSG 2, 236) ${ }^{8}$. Il décrit la modernité comme un processus général de «dissolution des substances en des fonctions, du solide et du durable dans le flux d'un incessant développement »(GSG 4, 330). Du point de vue épistémologique, il professe un nominalisme pragmatique: le concept scientifique est une « unité subjective » découpant un « objet de science » dans la multiplicité instable des phénomènes. La pertinence du concept dépend des fins que l'on se donne : elle est une « question de

\footnotetext{
${ }^{7}$ Contre l'équation heideggérienne humanisme = anthropologie, hâtivement posée et hâtivement reprise, nous considérons, avec Stéphane Toussaint, qu'il faut bien distinguer le problème de la "science de l'homme » et celui de la «culture de l'homme", le problème de la nature (ou de l'absence de nature) de l'espèce humaine et le problème de la culture de l'homme (de son humanitas) (voir S. Toussaint, Humanismes / Antihumanismes de Ficin à Heidegger, Paris, Les Belles Lettres, 2008, p. 31-45). Le lecteur verra que nous n'avons pas la prétention de déterminer une prétendue essence de l'humanisme et encore moins de contribuer à éclairer ce qu'il fut en tant que phénomène historique. L'emploi de ce terme aujourd'hui fort usé par des usages intempestifs et imprécis pourrait sembler discutable. Il nous a paru légitime d'une part parce que nous lui avons donné une fonction essentiellement polémique (pour marquer une opposition face aux tendances à la « réduction anthropologique »), d'autre part parce que notre usage rejoint celui qu'en faisaient, sinon directement Simmel, du moins plusieurs de ses contemporains (voir infra n. 39).

${ }^{8}$ Les références renvoient à Georg Simmel Gesamtausgabe, Francfort-sur-le-Main, Suhrkamp, 1989, 23 vol. (le vol. 24 est en préparation).
} 
praxis » (GSG 2, 129). D'une manière générale, tout ce que nous considérons comme une "unité » n'est qu'une stabilisation momentanée d'éléments en action réciproque. La "société », par exemple, n'est que « la somme de l'action réciproque de ses membres » (GSG 2, 130). L' «individu» est, psychologiquement, un ensemble de représentations en actions réciproques, sociologiquement, un "croisement de cercles sociaux» (GSG 2, 241) et, d'un point de vue historique et évolutionniste, un " point d'intersection des fils sociaux » constituant le « développement historique de l'espèce » (GSG 1, 238). Cette relativisation générale affecte également le concept $\mathrm{d}^{\prime}$ " homme»: la « théorie de l'évolution » n'autorise pas à affirmer « l'unité et la durée de l'homme ». Le concept d'homme n'est que l'hypostase d'un état de " relative stabilité de certains traits » dans une évolution biologique et historique où tout se trouve dans un «status nascens continuel » (GSG $4,45)$. Tout ceci conduit au « rejet de l'unité anthropologique » (GSG $4,123)$.

Une chose est établie dès 1890 qui ne sera jamais remise en question: il n'y a pas d'homme ou d'humanité en général. Un homme est un assemblage d'éléments impersonnels, jamais caractérisés comme spécifiquement humains, mais biologiques, historiques ou sociaux - et, plus tard, comme nous le verrons, culturels ou spirituels.

\section{Fonctions axiologique et épistémologique du concept d'humanité}

\subsection{Nietzsche : 1 'humanité contre la société}

Simmel va pourtant utiliser ponctuellement le concept d'humanité et cela précisément pour limiter (d'un point de vue épistémologique) et critiquer ( $d$ 'un point de vue axiologique) la perspective fonctionnaliste et relativiste qui l'a conduit à défendre ce qu'il nommera plus tard un «sociologisme extrême » (GSG 12, 391).

C'est dans la monographie Schopenhauer et Nietzsche (1907) que cette opération a lieu. Simmel écrit : «le point de vue social - sociohistorique, socio-psychologique, socio-éthique - qui n'est qu'un point de vue parmi d'autres [...] est devenu, à la fin du dix-neuvième siècle, le point de vue absolu ». Or, « la signification principale de la philo-

sophie de l'histoire de Nietzsche, c'est d'avoir brisé l'identification moderne de la société et de l'humanité, d'avoir reconnu des valeurs qui sont profondément indépendantes [...] de la forme sociale de 
l'existence humaine » (GSG 10, 359). La référence à l'« humanité » va accompagner la reprise de questions axiologiques oubliées par le point de vue sociologique. Et, comme «l'humanité n'a de réalité que par les individus qui la constituent »(GSG 10, 360), c'est l'individu qui va se retrouver porteur de ces valeurs «indépendantes [...] de la forme sociale de l'existence humaine » - individu qui, pour le Simmel sociologue, n'était qu'un " point d'intersection », un concept fonctionnel.

On devine la trace de deux grandes thèses critiques de Nietzsche : 1. la critique de la sociologie comme discipline nivelante, faisant disparaître le problème culturel de la valeur ${ }^{9}$; 2 . la critique des critiques évolutionnistes et utilitaristes de la morale qui croient avoir réglé le problème de la valeur en montrant que les valeurs ont une histoire et qu'il n'y a de valeur que là où il y a des intérêts (sociaux en particulier $)^{10}$. Il est très vraisemblable que la lecture de Nietzsche a conduit Simmel, qui a écrit en 1890 une sociologie fonctionnaliste (Über sociale Differenzierung (GSG 2)) et en 1893-1894 une critique historiciste, sociologique et psychologique radicale de tous les concepts et absolus éthiques (Einleitung zur Moralwissenschaft (GSG 3 et 4)), a une forme d'autocritique, manifeste dans le retour du problème de la valeur, en particulier à partir de la Philosophie de l'argent (1900) ${ }^{11}$.

Que Simmel va-t-il faire de l'individualisme nietzschéen ? Il n'est alors pas le seul à l'invoquer et l'on pourrait penser qu'il est conquis, après y avoir résisté, par un lieu commun de la Kulturkritik, l'affirmation de la valeur éminente de l'individualité dans une époque de nivellement et de mécanisation généralisée. Il le fait toutefois avec un net recul critique comme en témoigne sa mise en garde contre $l^{\prime}$ " eudémonisme subjectiviste des "Nietzschéens" ", avec leur individualisme égotiste et volontariste à la mode (GSG 10, 391). Selon Simmel, Nietzsche est un «moraliste » qui a «élevé le personnalisme à un idéal objectif ». Il fait, comme le christianisme, de la "personnalité accomplie [...] le porteur de valeur absolu à l'intérieur du monde» (GSG 10, 357). Or, cette valeur ne repose pas sur une affirmation subjectiviste de soi mais, comme nous allons le voir, sur des qualités objectives.

\footnotetext{
${ }^{9}$ Voir par ex. «Le crépuscule des idoles », in F. Nietzsche, CEuvres II, Paris, Robert Laffont, 1993, p. 1012.

${ }^{10}$ Voir par ex. « La Généalogie de la morale », in F. Nietzsche, CEuvres II, op. cit., p. 780 et 819-820.

${ }^{11}$ Simmel écrit son premier texte sur Nietzsche, "Friedrich Nietzsche. Eine moralphilosophische Silhouette », en 1896 (GSG 5, 115-129). L'année suivante, il fait une recension critique du Nietzsche-Kultus de Ferdinand Tönnies (GSG 1, 400-408).
} 
Simmel rappelle pourtant que l'individu, pour Nietzsche (comme pour lui), loin d'être un " atome », est une " erreur », une "fiction », la synthèse relative d'une multiplicité d'éléments (GSG 10, 360). Mais cette synthèse, précisément, est productrice de valeur. La multiplicité qu'enveloppe l'individu est celle de l'histoire; Simmel cite le $\S 33 \mathrm{du}$ Crépuscule des idoles: l'individu est «toute une lignée de l'homme jusqu'à lui-même », à partir de laquelle «la vie » peut faire "un pas en avant » (GSG 10, 360). L'interprétation de ce pas en avant de la vie en termes de valeur de l'humanité est autorisée par Nietzsche luimême, lorsqu'il nomme « humanité » le sentiment de l'individu «qui sait considérer l'histoire de l'homme [...] comme son histoire » et pressent, dans cette concentration de l'histoire en lui, l'horizon $\mathrm{d}^{\prime}$ « une noblesse nouvelle $»^{12}$.

Or, comme Simmel l'affirme dans sa recension du Nietzsche-Kultus de Tönnies, la «noblesse » est une valeur individuelle mais objective. Cette valeur a certes une genèse: "la noblesse a une dimension biologique, elle est l'effet d'un élevage sévère ». Néanmoins, elle est également une "qualité spirituelle», dotée d'une "valeur sui generis ", que nous reconnaissons par un "sentiment tout à fait spécifique »(GSG 1, 403-404). On observe ici une première séparation, dès 1897, des questions de genèse et de valeur. Penser en terme "d'humanité », chercher comme Nietzsche les conditions d'un « développement plus élevé de l'humanité » (GSG 7, 57), implique précisément de ne pas confondre la question anthropologique de la genèse de l'humain et la question axiologique de sa valeur. Cette lecture " humaniste » de Nietzsche permet à Simmel de maintenir une historicisation et une fonctionnalisation de l'être de l'homme sans tomber dans un relativisme dissolvant le problème de la valeur dans celui de l'utilité sociale ou biologique.

\subsection{L'absence d'unité des sciences de la culture}

C'est à présent vers le dernier chapitre de la Sociologie de 1908 qu'il faut nous tourner pour considérer le sens épistémologique de la distinction société/humanité. Il s'agit alors pour Simmel de différencier et de circonscrire l'approche sociologique par rapport à d'autres formes d'appréhension de la réalité socio-historique. $\mathrm{L}^{\prime}$ « humanité » n'est pas une réalité dotée d'une «conscience unitaire » ou en "développement continu», mais un "concept méthodique», une « idée ». Utiliser la catégorie d'humanité c'est adopter un «point de vue » distinct de celui qui est enveloppé par les catégories «société »,

${ }^{12}$ F. Nietzsche, «Le Gai Savoir », in CEuvres II, op. cit., p. 198. 
« individu » ou «nature » (GSG 8, 861). Ce n'est donc pas une catégorie englobant tout ce que l'on appelle habituellement les phénomènes humains, mais un point de vue spécifique sur ces phénomènes.

Ce point de vue consiste à considérer les «contenus de la réalité historique indépendamment de leur genèse et de leur signification sociale spécifiques » (GSG 8, 861). La description socio-historique de cette réalité est insuffisante. Les produits de l'histoire ont une genèse et des fonctions, mais il est possible de les considérer en eux-mêmes, abstraction faite de celles-ci. Cela revient à les traiter comme des " contenus » auxquels on peut attribuer signification et valeur par eux-mêmes, et non seulement comme des processus. C'est s'intéresser à la "culture objective ", à l' « esprit objectif » de la culture, esprit constitué par des structures économiques, des règles juridiques, des œuvres d'art, des théories scientifiques, etc. (GSG 8, 30) ${ }^{13}$. Le recours à la catégorie d'humanité est donc dirigé d'une part contre les prétentions de la sociologie à être une science globale de la culture, d'autre part contre l'historicisme qui dissout valeur et signification dans des processus génétiques ${ }^{14}$.

Simmel complexifie la distinction alors répandue entre sciences de la nature et sciences de l'esprit en distinguant clairement points de vue sur la société, sur l'histoire, et sur la culture. Si l'on ajoute à cela que la psychologie a un a priori bien distinct de ceux des autres sciences, on arrive à un paysage extrêmement fragmenté, sans horizon d'unification. À la division et à l'autonomie des différentes sciences ne correspond pas une partition ontologique du réel. L'objet de chaque science n'est pas une partie de la réalité mais l'ensemble de la réalité considérée sous une certaine perspective, mise en forme à partir d'un a priori spécifique. Ainsi l'échange peut-il par exemple être traité comme un fait économique, psychologique, historique ou esthétique (GSG 6, 11). Chaque science étant totalisante, il n'y a pas d'espace commun où leurs points de vue pourraient se compléter. Si une anthropologie est possible, elle sera donc une science parmi les autres, et non une synthèse de ces sciences.

\footnotetext{
${ }^{13}$ Sur la culture considérée comme esprit objectif, voir notamment la seconde partie du sixième chapitre de la Philosophie de l'argent (GSG 6, 620 sqq.), et D. Thouard, "Die Vergegenständlichung des Geistes. Simmels Hermeneutik der Objektivität», in Internationales Jahrbuch für Hermeneutik, no 9, Tübingen, Mohr Siebeck, 2010, p. 327-339.

${ }^{14}$ La philosophie de la culture doit permettre une « libération vis-à-vis de l'historicisme » (GSG $9,230)$ - historicisme dont l'essor fut en partie lié au projet anthropologique d'une «science de l'homme » (Hans Erich Bödeker, Göttingen vers 1800. L'Europe des sciences de l'homme, Philippe Büttgen et Michel Espagne (dir.), Paris, Cerf, 2010, Introduction, p. 11-14).
} 
Le projet anthropologique de dépasser la spécialisation scientifique pour construire une nouvelle image de l'homme ne pouvait donc guère trouver appui sur la théorie simmélienne de la connaissance. Il pouvait en revanche s'inspirer de la philosophie de la vie de Dilthey, qui a cherché à fonder l'unité des sciences de l'esprit dans la psychologie puis dans l'herméneutique, unité permise dans la mesure où

l'ensem-

ble du monde historique est considéré comme une expression ou une extériorisation de la vie des hommes (nous y reviendrons). Il pouvait également trouver appui sur la philosophie néo-kantienne de la culture pour laquelle l'humanité est une idée régulatrice, l'idée d'une «conscience culturelle» orientant vers un "système de la culture», une "vision du monde » vers laquelle convergeraient toutes les sciences ${ }^{15}$ (il est d'ailleurs significatif que le titre du dernier ouvrage d'Ernst Cassirer soit Essay on Man) ${ }^{16}$. On ne trouve en revanche aucun principe de fondation et d'unification des sciences dans l'épistémologie de Simmel : ni naturaliste, ni historique, ni psychologique, ni humaniste (au sens néo-kantien), pour reprendre les distinctions de Cassirer ${ }^{17}$.

\section{L'impossible réduction anthropologique de la culture}

Il existe néanmoins, en-deçà du problème de l'unité des sciences, une tension plus fondamentale entre la pensée de Simmel et le projet anthropologique. L'anthropologie n'entend pas simplement faire contribuer l'ensemble des sciences à une détermination de l'homme. Il s'agit également d'envisager sa destination. Kant l'a affirmé clairement: nous avons besoin d'une anthropologie car il faut, outre une " connaissance scolaire », une « connaissance du monde », c'est-à-dire une connaissance permettant d'orienter dans le monde. L'anthropologie philosophique, comme l'a montré Odo Marquard, est fidèle à cette impulsion : elle est un «tournant vers le monde de la vie » - qui est un monde culturel ${ }^{18}$. Aussi, quand elle aborde la question de la culture, ce n'est pas seulement pour élargir, selon un programme néo-kantien, la théorie de la connaissance de la nature à la

\footnotetext{
${ }^{15}$ Voir par ex. «Philosophie de la culture et idéalisme transcendantal », in W. Windelband, Qu'est-ce que la philosophie et autres textes, trad. É. Dufour, Paris, Vrin, 2002, p. 180.

${ }^{16}$ E. Cassirer, Essai sur l'homme, trad. N. Massa, Paris, Minuit, 1975.

${ }^{17}$ E. Cassirer, "Fondation humaniste et fondation naturaliste de la philosophie de la culture », in L'idée de l'histoire. Les inédits de Yale et autres traités d'exil, trad. F. Capeillères, Paris, Cerf, 1998. ${ }^{18}$ O. Marquard, "Zur Geschichte des philosophischen Begriffs "Anthropologie" seit dem Ende des achtzehnten Jahrhunderts ", in Schwierigkeiten mit der Geschichtsphilosophie, op. cit., p. 124.
} 
connaissance de la culture, mais pour interroger la fonction, pour la vie humaine, des différents domaines de la culture, afin de présenter l'image la plus complète possible de cette vie humaine. Il s'agit, selon le mot de Plessner, d'effectuer une « reprise dans la sphère d'activité de

l'hom-

me des exigences [...] qui lui sont imposées par les réalités religieuses, éthiques, juridiques, artistiques et scientifiques ». Le geste de Feuerbach, consistant à réduire la théologie à une anthropologie (inversée), reste un modèle de cette reprise ${ }^{19}$.

\subsection{L'autonomisation de la culture vis-à-vis de la vie}

Or l'impossibilité de cette reprise constitue une des thèses majeures de Simmel, une des rares thèses relativement connue, popularisée sous le nom de «tragédie de la culture ». Entre la Philosophie de l'argent de 1900 et la Lebensanschauung de 1918, elle connaît plusieurs versions, mais l'essentiel peut en être résumé comme suit ${ }^{20}$. Le processus historique de la culture est un processus d'objectivation. Sitôt que l'esprit s'objective, sous la forme de l'économie, du droit, de l'art, de la science, etc., il acquiert une forme d'indépendance vis-à-vis de l'activité spirituelle vivante qui l'a produit. Ainsi l'œuvre d'art contient-elle par exemple potentiellement plus de signification que ce que l'auteur a voulu y mettre et que ce que n'importe quel récepteur peut y trouver ${ }^{21}$.

Cette indépendance ne concerne pas seulement chaque produit de la culture pris isolément; elle est en fait un processus général d'autonomisation de la culture objective. Les formations culturelles ont d'abord une fonction: les propositions sur la réalité valent en tant qu'elles favorisent l'action (GSG 6, 101), l'échange économique est plus efficace que la rapine (GSG 6, 385), les formations religieuses expriment et renforcent les relations sociales (GSG 10, 64), etc. On peut rendre compte, de ce point de vue, de l'existence des formations culturelles en termes pragmatiques et évolutionnistes, voire biologiques. Mais l'objectivation fait émerger des lois objectives immanentes (logiques, économiques, religieuses, esthétiques, juridiques, etc.), indépendantes de la téléologie vitale. Ainsi les propositions sur la réalité apparaissent-elles porteuses d'une validité logique (GSG 6, 102) ; l'échange économique, en s'étendant, devient le lieu d'une norma-

\footnotetext{
${ }^{19}$ Sur les réserves de Simmel concernant cette opération, voir GSG 14, 374 et M. Amat, «Religion, mystique et crise de la culture à la fin de la période wilhelmienne. Perspectives sur Georg Simmel », in Revue de l'histoire des religions, 2015/3, p. 322.

${ }^{20}$ Pour une analyse plus précise, voir W. Geßner, Der Schatz im Acker. Georg Simmels Philosophie der Kultur, Weilerswist, Velbrück Wissenschaft, 2003, chap. 5.3.

${ }^{21}$ Voir par ex. GSG 16, 73.
} 
tivité indépendante des finalités extérieures à lui (GSG 6, 53) ; la religion se différencie de la société et devient une fin en soi (GSG 10, $117)$, etc. La dissociation des questions de genèse et de valeur est désormais clairement accomplie. Elle s'accompagne d'un désintérêt relatif pour les questions génétiques qui se manifeste par exemple par le peu de développements consacrés à la vie en tant que vie biologique. On peut toujours faire des hypothèses génétiques sur l'émergence de ce qui vaut, mais ce qui importe est que nous fassions l'expérience de tel ou tel type de valeur, et non les circonstances qui ont permis cette expérience et qui n'ont plus rien à voir avec elle.

Simmel nomme ce processus le «tournant vers l'idée », le passage à une orientation «idéo-centrée » de la vie (GSG 16, 244, 262). C'est à partir de là que l'on peut parler de culture stricto sensu, et pas seulement au sens du processus évolutif et historique spécifique qui singularise l'homme parmi les animaux. "Culture » a alors un sens axiologique et pas seulement descriptif. Il y a culture lorsqu'il y a expérience d'une valeur qui transcende la vie. Cette transcendance apparaît dans l'histoire, elle est, si l'on veut, relative. Mais elle est éprouvée comme s'imposant objectivement: ce qui vaut ne vaut pas moins parce qu'il a une genèse. Simmel recourt régulièrement à des images et à un lexique platonicien pour signifier cette validité objective de la culture. Dans la Lebensanschauung, il décrit ainsi la culture objective comme étant constituée de mondes différenciés «subsistant idéellement » et « indépendants, dans leur sens et leur valeur, de leur appropriation par des individus » (GSG 16, 238) ${ }^{22}$. La production de ces contenus dotés de sens et de valeur mobilise les énergies de la vie mais se déploie selon des logiques immanentes à la culture, et transcendantes par rapport à la vie. Ce déploiement d'une transcendance qui n'est pas métaphysique conduit Simmel à parler d'une "grâce de l'esprit objectif »(GSG 14,407); paradoxe d'un esprit que nous avons créé et qui dispense ensuite ses richesses à condition que nous nous soumettions à sa propre logique ${ }^{23}$.

\footnotetext{
${ }^{22}$ Simmel affirme, en suivant Hermann Lotze, que Platon aurait découvert « la catégorie du valide» (GSG 21, 911). Le relativisme de Simmel pourrait être considéré comme un type d'objectivisme sémantique, à côté de celui de Husserl, de Frege ou du premier Wittgenstein. On pourrait ainsi compléter la typologie des objectivismes sémantiques proposée par Jocelyn Benoist (« Variétés d'objectivisme sémantique", in Propositions et états de choses. Entre être et sens, J. Benoist (éd.), Paris, Vrin, 2006). Le relativisme philosophique a vocation, comme l'explique Simmel à Rickert en 1898, à rendre compte à la fois de la relativité de la valeur et de son objectivité (GSG 22, 292).

${ }^{23}$ Dans ses leçons de pédagogie de 1915-1916 (GSG 21), Simmel développe certaines des conséquences de cette transcendance fonctionnelle de la culture objective sur le processus éducatif. Sur ce point voir M. Amat, «La pédagogie sur ses deux jambes. Vie et esprit objectif dans les Leçons de pédagogie de Georg Simmel», in Différenciations et réciprocités. Regards simméliens sur la société
} 
La philosophie de la culture de Georg Simmel

contemporaine, D. Thouard et B. Zimmermann (éd.), Paris, CNRS, 2016 [à paraître]. Sur la nécessité, pour la philosophie de l'éducation, de reconnaître cette transcendance, voir Denis Kambouchner, L'école, question philosophique, Paris, Fayard, 2013, p. 96 sqq. et Laurent Jaffro, « Les objets de l'éducation : quelle ontologie? ", Revue de métaphysique et de morale, no 56, Paris, PUF, 2007/4, p. 429-448. 


\subsection{Simmel, Dilthey et Husserl}

La prétendue «philosophie de la vie» de Simmel se distingue nettement, en ce point, de celle de Dilthey, plus à même d'inspirer l'anthropologie philosophique. La description de la culture que l'on vient de proposer brise l'unité, chère à Dilthey, du "monde historique » ou " monde de l'esprit». "L'esprit, écrit-il, s'est objectivé dans ces réalités extérieures, des fins s'y sont forgées, des valeurs s'y sont réalisées [...]. Il y a ainsi entre moi et ces réalités une relation vitale. Leur caractère finalisé est fondé dans ma capacité de poser des fins, ce qu'il y a en elles de beau et de bien est fondé sur ma capacité $\mathrm{d}$ 'instituer des valeurs [...]. Même les concepts les plus abstraits sont des dérivés d'expériences vécues ${ }^{24}$. Simmel affirme au contraire une rupture de cette " relation vitale ». L'introduction du concept d'esprit objectif par Dilthey (dix ans après Simmel) ne le mène donc pas aux mêmes conclusions. Il s'agit bien pour Dilthey de reconnaître une autonomie des produits de la culture, et de ne pas rabattre de manière psychologiste toute signification sur une expérience vécue ${ }^{25}$. Toutefois, les objectivations restent fondamentalement des expressions de la vie. L'herméneute de la culture cherche à montrer leur cohérence et leurs relations afin de saisir un «ensemble vital historique ${ }^{26}$. Simmel rompt avec cet expressivisme.

Derrière ce "monde historique", c'est d'ailleurs de nature humaine qu'il s'agit pour Dilthey. On répète souvent sa fameuse formule : «ce qu'est l'homme, seule son histoire le dit». Mais cette histoire ne conduit pas à une relativisation radicale de l'idée d'homme. On peut même considérer, avec Marquard, que la philosophie de Dilthey, avant d'être un tournant vers l'histoire, est un tournant vers la nature, dirigé contre la dissolution nietzschéenne de l'homme dans l'histoire: malgré la diversité de ses expressions historiques, «la nature humaine [est] toujours la même $»^{27}$. Plessner est diltheyen lorsqu'il défend un "principe de relativisation de toutes les sphères intemporelles d'une civilisation par rapport à l'homme, considéré comme source à l'horizon de l'histoire», principe qui reprend celui de "réduction anthropologique ${ }^{28}$. Or, nous avons vu que ce à quoi

\footnotetext{
${ }^{24} \mathrm{~W}$. Dilthey, L'Édification du monde historique dans les sciences de l'esprit, trad. S. Mesure, Paris, Cerf, p. 72.

${ }^{25}$ Là-dessus, voir J.-C. Gens, La pensée herméneutique de Dilthey, entre néokantisme et phénoménologie, Villeneuve d'Ascq, Presses universitaires du Septentrion, 2002, p. 116-118.

${ }^{26}$ W. Dilthey, L'Édification du monde historique dans les sciences de l'esprit, op. cit., p. 105.

${ }^{27}$ W. Dilthey, «Die Typen der Weltanschauung », in Gesammelte Schriften VIII. Weltanschauungslehre, Stuttgart, Teubner, 1991, p. 79.

${ }^{28}$ H. Plessner, Gesammelte Schriften VI, Francfort-sur-le-Main, Suhrkamp, 1980-1985, p. 318.
} 
l'histoire nous donne accès ce ne sont pas seulement des expressions de l'homme, des valeurs ou des significations humaines. L'esprit objectif est en excès par rapport à l'homme. La connaissance de la culture est plus qu'une connaissance de soi de l'homme et la question « qu'est-ce que l'homme? » n'est donc pas le point de convergence de toutes les questions que peuvent poser la science et la philosophie.

De ce point de vue, Simmel apparaît plus proche de Husserl, qui $\mathrm{s}^{\prime}$ oppose à la relativisation anthropologique de l'expérience de la validité et de la signification, que ce soit d'une manière psychologiste ou historiste ${ }^{29}$. Or Husserl s'en prend précisément à Dilthey, en particulier dans son article "La philosophie comme science rigoureuse ", qui paraît en 1911 dans la revue Logos, et dont on sait qu'il sera lu avec attention et intérêt par Simmel ${ }^{30}$. Selon Husserl, s'il peut y avoir une "philosophie des sciences de l'esprit», celle-ci devrait reposer non sur la psychologie ou l'histoire (et finalement l'anthropologie) mais sur une "théorie phénoménologique des essences " ${ }^{31}$. Si ce vocabulaire ne saurait être celui de Simmel, on constate une proximité entre ce projet et celui d'une philosophie de la culture qui serait une théorie de l'esprit et de la valeur objectifs, distincte de la sociologie, de la psychologie, de l'histoire, ou de la biologie (et donc de l'anthropologie $)^{32}$. Ainsi faut-il par exemple - de manière quasiment phénoménologique - reconnaître la valeur comme un "fait originaire » (Urphänomen) faisant l'objet non d'un simple «sentiment», mais « d'une conviction, d'une affirmation, d'une reconnaissance » (GSG 6, 27). Les présupposés théoriques sont cependant bien différents de ceux de la phénoménologie husserlienne. Il ne s'agit pas, en particulier, de poser une conscience a priori, en position de fondement. $C^{\prime}$ est par une action réciproque indéfinie de la vie et de ses objecti-

\footnotetext{
${ }^{29}$ Sur ce point, voir par exemple J.-C. Monod, " "L'interdit anthropologique" chez Husserl et Heidegger et sa transgression par Blumenberg ", Revue Germanique Internationale, $\mathrm{n}^{\circ} 10$, en ligne, 2009 , p. 222.

${ }^{30}$ Comme en témoigne la lettre à Husserl du 13 mars 1911 (GSG 22, 950). L'introduction de la Philosophische Kultur, écrite quelques mois plus tard, et dans laquelle Simmel distingue la philosophie comme science et la «culture philosophique » répond d'ailleurs à la distinction établie par Husserl entre philosophie comme science et philosophie comme « conception du monde ». Simmel sera le principal contributeur de LOGOS entre 1910 et 1918, au point de l'avoir parfois considéré comme «son journal» (R. Kramme, «Philosophische Kultur als Programm. Die Konstituierungsphase des LOGOS », in Heidelberg im Schnittpunkt intellektueller Kreise, H. Treiber (éd.), Opladen, Westdeutscher Verlag, 1995).

${ }^{31}$ E. Husserl, La philosophie comme science rigoureuse, trad. M. de Launay, Paris, PUF, 1989, p. 68.

${ }^{32}$ Conjuguant les influences de Husserl et de Simmel, Scheler est sur la même ligne dans le Formalisme en éthique (1916) : «il faut rejeter avec fermeté [...] toute doctrine qui fait dépendre l'être-axiologique de la structure humaine ", sous peine de tomber dans " l'idole de la connaissance de soi », travers de l'anthropologie (cité par O. Agard, «La question de l'humanisme chez Max Scheler », Revue Germanique Internationale, nº 10, en ligne, 2009, p. 166).
} 
vations qu'émerge l'expérience de la valeur comme validité. En outre, s'il arrive à Simmel d'utiliser le concept d'humanité comme marqueur de la question de la valeur, celui-ci ne joue jamais le rôle d'une idée régulatrice, comme c'est le cas non seulement chez les néokantiens, mais aussi chez Husserl. Le « développement de la culture » ne peut donc être décrit, comme une "réalisation progressive de l'idée éternelle de l'humanité ", ainsi que le fait Husserl dans La philosophie comme science rigoureuse ${ }^{33}$. La résistance de Simmel à une anthropologie naturaliste ou historiste n'ouvre donc pas la possibilité d'une anthropologie transcendantale ${ }^{34}$.

\section{Le problème de la « culture individuelle »}

La rupture de la relation d'expressivité entre la vie et l'esprit objectif entraîne-t-elle l'impossibilité de toute synthèse? Non bien sûr : chaque homme, en tant qu'être de culture, constitue un certain type et un certain degré de synthèse entre ces principes hétérogènes. Mais la synthèse est partielle, relative, conflictuelle. Elle ne saurait prendre la forme d'une unité culturelle retrouvée, vision du monde ou image de l'homme. Et, surtout, la charge de la synthèse, dans une modernité hautement différenciée et objectivée, repose essentiellement sur les individus. Lorsque cette synthèse de vie et d'esprit objectif est pensée non seulement comme un fait anthropologique (l'homme est un être de culture), mais comme une forme d'accomplissement individuel, on parle de "culture individuelle». La culture prend alors le sens classique d'un « accomplissement de l'âme » atteint «en faisant le détour par les formations du travail spirituel et historique de l'espèce »(GSG 16, 37). Elle est la «synthèse [particulièrement réussie et harmonieuse] d'une évolution subjective et de contenus spirituels objectifs » (GSG 14, 399). Or une telle synthèse est fondamentalement problématique, comme l'indique l'essai de 1911, Le Concept et la tragédie de la culture:

Les contenus grâce auxquels le moi doit accomplir cette organisation en un monde unifié qui lui soit propre, n'appartiennent pas seulement à lui; ils lui sont donnés, à partir d'un au-dehors spatial, temporel, idéel; ils sont en même temps les contenus d'autres mondes sociaux ou métaphysiques, conceptuels ou éthiques. Dans ces mondes ils possèdent des formes et des corrélations entre eux qui ne veulent pas coïncider avec celles du moi.

\footnotetext{
${ }^{33}$ E. Husserl, La philosophie comme science rigoureuse, op. cit., p. 76.

${ }^{34}$ Voir L. Perreau, Le monde social selon Husserl, Berlin, Springer, 2013. p. 314 sqq.
} 
L'homme se tient [...] à l'intersection de plusieurs sphères de valeur et de puissance objective, [...] de telle sorte qu'il est pour ainsi dire l'intersection de lui-même et de sphères d'exigences étrangères (GSG 14, 404).

L'homme est un être dualiste, composé d'éléments hétérogènes. $\mathrm{D}^{\prime}$ un point de vue ontologique on peut dire qu'il est une réalité de second ordre. Formellement, l'homme reste l'unité relative et fonctionnelle constituée d'éléments impersonnels, qui était décrite dès 1890. Mais les cercles sociaux sont devenus des mondes idéels dont les contenus impersonnels sont dotés de sens et de valeur. La culture individuelle est ainsi une organisation et une animation de ces contenus dans la forme d'une vie individuelle. On voit Simmel proposer ici sa version $\mathrm{du}$ personnalisme qu'il attribuait à Nietzsche. L'immanentisme nietzschéen est complété par une théorie de la valeur et de l'esprit objectifs : Nietzsche a bien compris que la vie était « plus que vie »(Mehr-Leben), mais il a ignoré qu'elle était aussi «plus que la vie » (Mehr-als-Leben) (GSG 16, 229). La vie seule, même conçue comme auto-dépassement, ne saurait être un support de valeur ${ }^{35}$.

Ce texte révèle toutefois un autre legs : celui de l'humanisme et du romantisme allemand ${ }^{36}$. On constate en effet que la culture individuelle est décrite en termes de relation et tension entre l'individu et le monde, les deux termes de la relation ayant vocation à constituer des totalités et à s'assimiler l'un l'autre. C'est une façon d'appréhender la question de la culture, ou de la formation, directement liée à ce que l'on peut trouver chez Willhelm von Humboldt ou Schleiermacher. Il s'agit en effet, pour ce que l'on appelle, depuis Paulsen, le néo-humanisme allemand, de dépasser la conception intellectualiste de la culture héritée des Lumières en mettant au premier plan l'individualité vivante, par une sorte de retour à Leibniz. Toutefois, celle-ci ne pouvant se former de manière purement immanente, elle trouve son contenu dans un monde, qu'elle doit s'approprier pour l'exprimer ou le symboliser. Ainsi la formation consiste-t-elle, selon Humboldt, à "se saisir d'autant de mondes que possible ", afin de produire une « action réciproque entre notre moi et le monde ». Et c'est par l'intégration de la richesse "non-humaine" du monde que l'on peut " donner au concept d'humanité un contenu aussi grand que possi-

\footnotetext{
${ }^{35}$ On notera que dans sa critique des philosophies prétendant fonder la valeur sur la vie, Heinrich Rickert s'en prend à Nietzsche, à Dilthey, à la "philosophie biologiste ", mais pas à Simmel ("Valeurs de vie et valeurs de culture", in H. Rickert, Le système des valeurs et autres articles, trad. J. Farges, Paris, Vrin, 2007.

${ }^{36}$ Simmel rapproche d'ailleurs régulièrement l'individualisme de Nietzsche de celui de Goethe et des « romantiques» (Schleiermacher en particulier) (voir par ex. GSG 7, 52).
} 
ble» en chacun de nous ${ }^{37}$. L'humanité est bien un concept axiologique désignant la formation vivante de la matière $\mathrm{du}$ monde dans la forme de la vie individuelle. Un siècle plus tard, le monde est dit « culturel » et se décline au pluriel. La tension individu/monde est compliquée par celle entre les différents mondes, entre différents types de « systèmes de valeur» (science, arts, économie, droit, etc.) (GSG 15, 21) ${ }^{38}$. Simmel reste néanmoins fidèle à l'idée d'un enveloppement réciproque de l'individu et du monde pour penser la formation de l'homme : il s'agit à la fois de faire monde en soi et d'être une partie du monde, et de différents mondes. Ainsi se trouve reformulé, et adapté à une culture objective fonctionnelle et différenciée, un motif central de $l^{\prime}$ humanisme allemand ${ }^{39}$.

\section{Remarques conclusives : humanisme et réduction anthropologique}

Les mots $\mathrm{d}^{\prime}$ « homme » ou d' « humanité » ne désignent donc pas, chez Simmel, les objets problématiques d'une connaissance anthropologique à constituer, mais indiquent un problème axiologique avant d'être scientifique, celui de la culture conçue comme formation. Et encore ce problème n'est-il pas entièrement enveloppé par le concept d'homme: l'impossibilité de la réduction anthropologique signifie que l'on ne peut penser la formation de l'homme sans le mettre en relation avec autre chose que lui. Cet autre, la culture objective, est

\footnotetext{
${ }^{37} \mathrm{~W}$. von Humboldt, De l'esprit de l'humanité et autres essais sur le déploiement de soi, trad. O. Manonni, Paris, Premières Pierres, 2004, p. 29-30. Traduction modifiée.

${ }^{38}$ Sur la différenciation de la culture en «mondes », voir M. Amat, « Kulturphilosophie als Kosmologie. Der Beispiel Georg Simmels », Zeitschrift für Kulturphilosophie, 2015/1-2.

${ }^{39}$ Motif qui peut être vu comme une nouvelle version du thème de la correspondance entre microcosme et macrocosme qui caractérise, si l'on en croit Cassirer, la philosophie de l'humanisme renaissant (Individu et cosmos dans la philosophie de la Renaissance, Paris, Minuit, 1983, (trad. Pierre Quillet), voir en particulier p. 241). Sur ce point voir également Richard Hönigswald, «Philosophische Motive im neuzeitlichen Humanismus», Kant-Studien, $\mathrm{n}^{\circ}$ 25, 1922. L'« humanisme moderne » se caractérise entre autres, selon l'auteur, par l'émergence de « différents domaines de validité » auxquels correspondent des « formes autonomes de la conscience culturelle » (ibid., p. 2930). Dans ce contexte la culture est l'« idée d'un système de valeurs se réalisant dans les personnes, dans la mesure où cette réalisation (Verwirklichung) est elle-même considérée comme une valeur objective " (ibid., p. 32) ; la culture, comme processus individuel et historique, est un "nouveau domaine de validité objective » (ibid., p. 34). Dans le contexte de cet «humanisme philosophique» qui est en même temps une "renaissance de Platon », la culture comme synthèse entre "sujet » et « objet », « individu » et "système de valeurs », est décrite en termes mystiques (chez Ficin par exemple) (ibid., 38-39).
} 
certes anthropologiquement déterminé d'un point de vue génétique mais sa valeur n'est pas susceptible d'une réduction anthropologique.

Ce n'est donc pas la caractérisation de l'homme (être problématique, excentré, etc. $)^{40}$ qui éloigne Simmel de l'anthropologie philosophique mais l'articulation de cette caractérisation à une philosophie de la culture fidèle à une orientation humaniste. L'anthropologie philosophique aura tendance à se crisper sur l'absence d'être de l'homme, qui apparaîtra comme une pure négativité, une déficience ontologique. Cette déficience affectera alors l'ensemble de la culture puisque, en vertu du principe de réduction anthropologique, la valeur de celle-ci est indexée sur celle de l'homme. Ainsi Arnold Gehlen tendrat-il, à partir de sa fameuse caractérisation (empruntée à Herder) de $l^{\prime}$ homme comme " être lacunaire », à réduire la culture à l' « univers compensatoire de la technique et des institutions ${ }^{41}$, « retourn[ant] les prémisses humanistes [...] de Herder dans un sens autoritaire et répressif ${ }^{42}$. Cette indétermination de l'homme, pensée non seulement comme capacité d'être modelé, mais aussi comme une véritable indétermination ontologique, est pourtant déjà admise par l'humanisme renaissant (que l'on pense à l'homme pichien, " ni terrestre ni céleste, ni mortel ni immortel » ${ }^{43}$ ) sans qu'en soient tirées des conclusions pessimistes ou tragiques. L'absence de réduction anthropologique préserve justement l'humanisme italien, puis allemand, d'une interprétation tragique de cette indétermination: l'être déficient qu'est l'homme a hors de lui une réalité porteuse de valeur (Dieu, le monde, la culture...), en relation avec laquelle il peut prendre forme et consistance. Chez Simmel, la transcendance (fonctionnelle) de l'esprit objectif offre une nouvelle figure de cette réalité objective. Cette transcendance favorise certes les phénomènes de réification et d'aliénation de la vie, mais elle est la condition de l'objectivité de la valeur. Ce qui est «tragédie de la culture » sous une certaine perspective est " grâce de l'esprit objectif » sous une autre.

Le problème décisif, si l'on s'intéresse à la formation et à la valeur de la vie humaine, n'est donc pas, comme on l'a répété à satiété, que l'homme n'ait plus de "nature »; en a-t-il jamais eue ? trouve-t-on si

\footnotetext{
${ }^{40}$ Voir par ex. le concept de «positionalité excentrique » chez Plessner (Gesammelte Schriften IV, op. cit., p. 364).

${ }^{41}$ É. Bimbenet, Ch. Sommer, «Les métaphores de l'humain. L'anthropologie de Hans Blumenberg ", Le débat, no 180, Paris, Gallimard, 2014/3, p. 92.

${ }^{42} \mathrm{M}$. Marino, "L'Anthropologie de la "créature déficiente" [Mängelwesen] et la question de l'origine des langues : chemins de Gehlen vers Herder », Revue Germanique Internationale, $n^{\circ} 10$, en ligne, 2009, p. 187.

${ }^{43}$ Voir S. Toussaint, Humanismes / Antihumanismes de Ficin à Heidegger, op. cit., p. 43.
} 
souvent, dans l'histoire des idées, des déterminations de la «nature humaine»? y a-t-il des époques où l'homme n'ait pas été «un problème pour lui-même $»^{44}$ ? Si nous avons le sentiment de traverser, depuis plus d'un siècle, une «crise de la culture » d'une gravité

particulière, dont la dévaluation ou le discrédit des idées dites "humanistes " est l'un des symptômes, c'est sans doute plus à cause d'une perte de valeur du monde qu'en raison de la prétendue découverte du manque d'être de l'homme. Insister sur la transcendance relative de la culture est précisément un moyen de redonner de la valeur au monde. Simmel a voulu le faire tout en assumant pleinement le tournant relativiste de la modernité, en proposant une théorie relativiste mais non nihiliste de la valeur.

Redonner une valeur objective aux produits de la culture en refusant la réduction anthropologique, c'est enfin rééquilibrer le discours d'une modernité philosophique pour laquelle «au commencement était l'action ». La philosophie transcendantale, la philosophie de la vie, puis l'anthropologie allemande, décrivent l'homme essentiellement comme un être d'action et de production, un homo faber. Or, en décrivant la formation comme une appropriation de contenus objectifs, sans lesquels l'homme ne saurait trouver de consistance, Simmel redonne une certaine légitimité aux métaphores platoniciennes ou stoïciennes de la possession et de la contemplation ${ }^{45}$. La culture est évidemment activité, mais elle est aussi avoir. Cette orientation de la pensée la pensée de Simmel peut également être vue comme une résistance par anticipation aux diverses formes de l'existentialisme, qui substituent à la figure de l'homme cultivé (un homme plein d'autre chose que de lui-même), celles de l'homme héroïque, résolu, engagé, etc. - ou celle d'un homme invité à approfondir le néant qu'il est plutôt qu'à chercher à le remplir et à lui donner forme.

\footnotetext{
${ }^{44}$ M. Scheler, La situation de l'homme dans le monde, Paris, Aubier-Montaigne, 1951, p. 16.

${ }^{45}$ Sur ce point, voir R. Konersmann, «Metaphern für Kultur», in Handbuch Kulturphilosophie, Konersmann (éd.), Stuttgart/Weimar, Metzler, 2012, p. 429-430.
} 